\title{
Las prácticas corporales y la educación Física en la escuela primaria entre 1870 y 1913
}

\section{Claudia Ximena Herrera Beltrán*}

El trabajo que a continuación se presenta, corresponde a los resultados de la investigación histórica sobre las prácticas corporales y la Educación física en la escuela primaria en Colombia a finales del siglo XIX y comienzos del siglo XX. Voy a dar cuenta, primero, del lugar que ocupó el cuerpo dentro de los objetos de saber pedagógico; segundo, a reconstruir una historia del cuerpo al interior de la pedagogía como práctica de saber; y tercero, a rastrear los comienzos de la Educación física y su relación con las prácticas corporales escolares.

\section{Como y desde donde se construye el cuerpo infantil en la modernidad}

En los 43 años que abarca este estudio, transcurridos entre 1870 y 1913, el cuerpo infantil fue objeto de diversas prácticas escolares que contribuyeron a configurar el saber de la Educación física, situándola al lado de otras ramas de instrucción en el lugar que le correspondía como parte fundamental en la educación integral del niño colombiano.

Así, la escuela colombiana del siglo XIX, institución impuesta desde Europa, fue un espacio privilegiado para la enseñanza desde tina práctica pedagógica de saber y de poder, por el cual circularon discursos y teorías, normas y leyes que fueron construyendo el cuerpo infantil. Este conocimiento, objeto del saber pedagógico, de la misma forma que lo fueron el maestro, el niño, la instrucción y la escuela, entre otros y que discurrió en documentos dc prensa, en revistas y en libros, permitió mostrar y analizar las condiciones de posibilidad del cuerpo, dentro dc la práctica pedagógica.

Un acercamiento a los antecedentes políticos sociales y económicos de la época, permite afirmar que fue de grandes conflictos, entre los que se cuentan la disputa por el poder entre los partidos políticos liberal y conservador, su posición frente al poder de la iglesia y la dirección de un sistema nacional de enseñanza que llegara a los lugares más apartados de la nación, para alcanzar el progreso y la civilización que tan afanosamente se deseaba.

El Decreto Orgánico de instrucción Pública Primaria DOIPP de 1870, le asignó a la escuela como su objeto principal, la formación de hombres sanos de cuerpo y espíritu, dignos y capaces de ser ciudadanos y magistrados de una sociedad republicana y libre. De allí que la educación debió moldear buenos ciudadanos, capaces de defender a la patria, rectos moralmente y con un carácter fuerte y estable, propiciando, a la vez, el desarrollo de la ciencia y la renovación de las sociedades. Por otra parte, también se educaban hombres ilustrados que basaban su conocimiento en Dios y en las auténticas fuentes de la religión católica.

En medio de grandes discusiones políticas y sociales alrededor de cómo debía ser la educación, fue claro para todos que sólo a través de ella era posible alcanzar el progreso y los ideales de una nación moderna. Desde el momento en el cual la instrucción pública fue asumida, organizada y controlada por el Estado, la polémica que se dio hasta 1886 estuvo centrada en definir si debía tener o no la injerencia directa de la pedagogía católica

\footnotetext{
* Exalumna Magíster en Historia de la Educación y la Pedagogía. Universidad Pedagógica Nacional. 
y estar o no en concordancia con los preceptos de esa religión. ¿Se debía enseñar la moral católica y la moral civil, o sería la enseñanza de la moral católica un asunto personal y un derecho a la libertad de culto impartida fuera de la escuela, mientras en su interior se enseñaba una moral civil como beneficio social necesario?

La polémica antes descrita llegó a su fin en 1886, cuando el gobierno de Núñez, mediante el Concordato, le entregó la organización y dirección de la instrucción pública a la iglesia católica. De esta forma su pedagogía, como un poder moral fuertemente arraigado en nuestra sociedad, fue configurando las prácticas escolares de manera singular.

El saber como objeto de la práctica pedagógica albergó diferentes discursos que fueron construyendo un modo de ser del pensamiento educativo de la época. Entre ellos aparecen el pensamiento pedagógico del catolicismo, las ideas de Lancaster, el pensamiento de Rousseau, la corriente prestalozziana y la froebeliana, sobre las cuales se fundó la pedagogía colombiana.

El poder del catolicismo, tan arraigado en nuestra cultura, en el período de investigación, y amparado por los partidos políticos y autorizada por la legislación, penetró sistemática, estratégica y totalmente en la instrucción pública colombiana mucho más allá de 1913 - en 1 932, retornó al Estado el control de la educación-. Gracias a su autoridad, obtuvo posiciones importantes en todos los estados confederados en que estuvo dividido el país basta 1886. Tenía en su poder prohibir textos, declarar y perseguir a los enemigos fuesen estos ateos, masones, benthamistas o liberales. En la escuela se impuso el catecismo y vigiló y censuró a los maestros en la medida en que su actuación pudiera llevar a la depravación de los niños y por ello a la ruina de la sociedad. Se opuso antes y después del Concordato a todas aquellas doctrinas y enseñanzas contrarias al dogma católico. Desde el púlpito animó al pueblo a pronunciarse violentamente contra la influencia protestante que estaban recibiendo los alumnos de las escuelas normales a cargo de un grupo de profesores alemanes traídos por el gobierno liberal. El conflicto que concluyó, dolorosamente, con la guerra de las escuelas en 1876, en la que se destruyeron algunas instituciones escolares y se asesinaron varios maestros encargados.

La moral católica fue viabilizada en la escuela por medio del cuerpo infantil desde prácticas corporales estrictas y repetidas. Así fue posible imbuir a los niños en principios católicos como la conversión del alma, la verdadera fe que no da espacio a la duda y la búsqueda de la perfección desde disciplinas impuestas que lo acercaran a la imagen de Dios. Dichas prácticas se hicieron desde el cuerpo como muestra de la distancia que se podía tomar de él y de la carne, ya que esto era lo que perdía al hombre. Se trató, entonces, de controlar y de dominar al cuerpo, desde las virtudes que se alcanzan por el hábito de la obediencia y la atención como consecuencia directa de la pedagogía divina.

Según estos preceptos divinos, se reeducaba al hombre pecador rehabilitándolo desde el temor y el amor a Dios. Una pedagogía católica que consideraba al hombre una unidad de cuerpo y alma, la que sólo podía ser comprendida o conocida así misma a la luz de un Dios, que está fuera y por encima del hombre. Para el catolicismo, la función teológica del cuerpo humano procedía de su existencia corno morada del alma y a la vez su cárcel. El cuerpo era servidor del alma y mediador entre ella y el mundo. El cuerpo obedecía o desobedecía, era el portador del deseo y del agente realizador, permitía la exteriorización del pensamiento, del sentir y del querer, trazaba una frontera con los otros, expresaba su individualidad y permitía la relación social con los otros. Todo se conjugó de manera que 
el papel del cuerpo se hizo necesario en el desarrollo personal de la razón, de la previsión y del propósito moral.

La manera como la carne se salvaba desde el cuerpo, era por medio de los ejercicios de ascetismo, de control, de dolor, de ayuno y de disciplina. No se le abandonaba, se le conocía para renunciar a ella. Para el catolicismo no fue lícito despreciar la vida corporal, ya que la profunda integración de ella con el alma permitía alcanzar la comunicación con la divinidad. Los azotes, como decía Loyola, eran para dominarlo, no para castigarlo. De allí la afirmación de que el catolicismo antes que despreciar el cuerpo privilegió su cuidado para controlarlo.

Este pensamiento pedagógico se mezcló con otras corrientes e ideas como las de Rousseau, Lancaster, Pestalozzi y Froebel, que llegaron al país en publicaciones traídas por algunos intelectuales que arribaron de Europa.

Revisados algunos documentos en que se habla de la obra de Rousseau, se puede afirmar que la lectura de su obra en Colombia a finales del siglo XIX y comienzos del XX, no se realizó en forma completa y profunda, pues las ideas que más se mencionaban fueron aplicadas, en muchos casos, de forma contraria a como las planteó Rousseau. Por ejemplo, la educación con un preceptor por alumno, para formarlo lejos de la influencia nefasta del mundo, no fue posible en Colombia, pues la instrucción pública, en su afán por educar a la mayoría, se vio obligada a confinar en el mismo lugar a cientos de niños para intentar cumplir con su rápida educación en el mismo tiempo. Se dio entonces una homogenización de las respuestas infantiles contestando todos en coro, moviéndose todos al tiempo o silenciándose para ser buenos ciudadanos.

En cuanto a su idea de educar siguiendo el modelo de la naturaleza - todo individuo posee una naturaleza inherentemente buena, la que contiene todo el potencial necesario para alcanzar la perfección humana, primero por medio de la labor de la madre y después del preceptor-,en el país, no fue viabilizada debido a la incipiente formación que tenían los pocos maestros con que se contaba en aquella época. De forma parecida, la idea de educar moralmente al niño, sólo a partir de los 16 años, junto con una elección personal de la ideología religiosa a seguir, generó un fuerte rechazo por parte de la iglesia y de los que veían en la educación moral religiosa.

Asimismo, el planteamiento de Rousseau de educar desde el hábito, y que fue también una de las propuestas de la pedagogía católica, se siguió sólo en parte, pues la idea de romper con toda actividad en el momento en que el preceptor así lo quería, sólo por el hecho de ser él quien lo decidiera, no se desarrolló, pues se seguían los preceptos católicos Como verdades inmutables e incuestionables que no atendían al capricho del preceptor.

A diferencia de lo expuesto anteriormente, la vigilancia permanente sobre el cuerpo y sobre la imaginación del niño que propuso Rousseau y que obligaban a mantenerlo ocupado, fue herramienta muy útil en la educación del país a la hora de evitar que la infancia se perdiera en vicios que pudieran causarle la muerte y la llevaran al crimen. De igual manera, la relación de dependencia entre el alumno y el preceptor, que recorre toda su obra, fue una de las características de la educación colombiana, en la medida en que el niño fue considerado carente, incompleto y débil, y el maestro era quien con su saber debía conducirlo por el camino del bien. 
Educar, atendiendo cuidadosamente a las etapas por las que transitó el niño, como lo proponía el pensamiento rousseauniano, no tuvo lugar en la educación colombiana, pues la organización de los grupos escolares por edades se dificultó, durante el período de investigación. Además, se educó desde la obediencia total, que no planteó —por lo menos de forma escrita - como propósito la conciencia propia del niño frente al acto educativo de que era objeto.

En el plano histórico, las apropiaciones que tomo la educación de la corriente lancasteriana se remontan al gobierno de Bolívar y se deben a su afán por educar a la mayor cantidad posible de niños con pocos maestros, ayudados por monitores que estaban a su cargo. El método de enseñanza mutua, como se le denominó, hizo visible el cuerpo de los niños en la medida en que éste fue objeto tic registros precisos, cuantitativos y cualitativos, que facilitaban la labor de los monitores. Estos eran los niños mayores y más adelantados, a quienes se les asignaban tareas menores como controlar la disciplina, comprobar la asistencia, tomar las lecciones y revisar las tareas, todo bajo la vigilancia del maestro encargado.

Este sistema favoreció la mirada de todos sobre todos, sobre las acciones del cuerpo infantil, y sobre el aprovechamiento del tiempo dentro del espacio escolar. Así, la disciplina y la obediencia a las normas y a los buenos modales fueron objetivos de todos. Sin embargo, esta enseñanza mutua tuvo sus detractores, quienes insistían en que una educación en manos de monitores inexpertos no era una buena educación, la que además restaba al profesor el poder que la pedagogía le había otorgado, un poder considerado incuestionable en razón a su edad, su saber y su experiencia.

De Pestalozzi se tomó la idea del desarrollo armónico de todas las facultades físicas, morales e intelectuales del niño desde la experiencia directa con la naturaleza, por medio de su método de las lecciones de cosas. Un método que se valía de la acción permanente del cuerpo del niño, con el mundo, que era por donde entraban las intuiciones. Así, plantear que la educación intelectual entraba también por la observación del mundo, que hacían los sentidos, posibilitó pensar el cuerpo como ayuda para el desarrollo de las facultades intelectuales desde el desarrollo de las facultades físicas. Esto apoyó también a la pedagogía católica en la medida en que colocó al cuerpo en el primer escalón del conocimiento del mundo y en la medida en que actuaba como mediador entre el alma y el mundo exterior desde los sentidos.

La apropiación, en Colombia, de las teorías educativas pestalozzianas, no profundizo sobre el estudio y el desarrollo de lo corporal en el niño, pues aunque se habló del desarrollo de las facultades físicas y de su relación con las facultades intelectuales, la actividad física, sólo llegó a considerarse como descanso a la actividad intelectual del niño que iba a la escuela.

La educación colombiana tomó de Froebel, su interés por el juego, desde el jardín de infantes, como la manera privilegiada de conocer su propio cuerpo, su movimiento y de aprender a trabajar; lo mismo que el valor de la educación por parte de las madres a los niños. Sin embargo, la libertad propuesta por Froebel se contradice con sus propios principios, en la medida en que va decidiendo cuáles deben ser los juegos más apropiados para cada edad, coartando la libre selección de los mismos por los niños.

Estas ideas pedagógicas, que fueron construyendo el campo teórico sobre el cual se fundó la pedagogía colombiana y la Educación Física, aparecieron en los discursos pronunciados en los colegios, en conferencias y en clases, y fueron publicados por la 
prensa oficial y por la privada, atendiendo en sus diversos momentos a unas concepciones y a un ideal educativo singular.

Así, a la pedagogía, considerada como el arte de guiar al niño, se le asignó posteriormente la categoría de teoría de la educación, ocupada de los métodos, principios y medios necesarios para la enseñanza. Y la enseñanza fue a su vez la acción y el arte encomendados al institutor, y fue, también, la suma de todo aquello que ocurría en la escuela; de tal forma que entre sus componentes estaban el alumno, el maestro y la enseñanza misma -los conocimientos y las habilidades-. La educación, fin último de la enseñanza, fue definida como la única fuente de riqueza del mundo, la que atendía al cultivo y desarrollo progresivo de las facultades humanas, dirigiendo al niño desde su cuerpo, su entendimiento y su corazón hacia la práctica de las virtudes -mente sana en cuerpo sano-. Educar, que no fue lo mismo que instruir, pues era un medio educativo que proporcionaba las herramientas y los conocimientos para permitirle al hombre valerse por sí mismo, siendo hábil y capaz, debió hacer del niño débil, ignorante e impotente, un hombre fuerte, honrado, laborioso, instruido según su posición y capaz de ser para él mismo, para su familia y para la patria, lo mejor y más útil posible. De esta manera, la educación del cuerpo fue pasando de apoyar a la educación intelectual a apoyar y lograr la educación moral, para ocupar, al final del período de investigación, casi el mismo lugar que las otras educaciones, por lo menos en lo que se refiere a los discursos.

La escuela en el siglo XIX fue impuesta en el país desde un cruce de fuerzas que le dieron posibilidad de existir y luego, de ser necesaria. El ordenamiento de que fue objeto la práctica pedagógica en su interior hizo posible aprender, vigilar, normalizar y educar. La institución educativa con características, tiempos y espacios propios también se ocupó del cuerpo de los nimios. En la escuela, el cuerpo de los mimos ingreso a un mecanismo de poder que lo exploró, lo desarticuló y lo recompuso. Esta nueva construcción corporal, encaminada hacia la docilidad y la utilidad, se dio gracias a la diversidad de prácticas escolares encaminadas a encauzar la conducta infantil. Se trataba de construir cuerpos dóciles y útiles.

La infancia, su idea y sus sentimientos heredados de Europa desde el siglo XVIII, fueron apropiados por la práctica pedagógica colombiana. El saber acerca del niño se movió, entonces, entre la inocencia, la debilidad, la incompletud, la fragilidad de cuerpo y alma y la manera como estas características debían educarse. Por un lado, se buscaba protegerlo de todo aquello que pudiera restarle inocencia, y por otro, encauzarlo por el camino del bien, de forma severa y disciplinada, por medio de prácticas corporales encaminadas a garantizar su salud, su higiene, su utilidad y su educación moral. El niño, a quien también se le consideró poseedor de todas las fuerzas inconmensurables de la humanidad, debía ser conducido hasta su perfecto desarrollo, dañado tal vez por vicios de conformación, para hacer de él un ser equilibrado que transmitiera todo eso a una nueva, más fuerte, bella, inteligente y virtuosa generación. Esta conducción de la infancia hacia el progreso y el mejoramiento de la raza tuvo que orientarse desde la acción y el movimiento corporal incesante, mediante el cual podía desarrollarse plenamente.

La educación del niño, en la escuela, privilegió el control, la vigilancia y el castigo sobre el cuerpo, como la forma de encauzar la conducta y enseñar a trabajar y a obedecer. Así, el panoptismo, como estrategia de control, encontró en la institución educativa un lugar privilegiado de realización.

El cuerpo y el alma, como los dos lados de la manifestación humana, se prestaban mutua influencia. El alma recibía las impresiones por medio del cuerpo y el cuerpo decidía 
no sólo de las facultades mentales del individuo, sino también de su carácter y de su porvenir. De allí la preocupación de educar y cultivar el cuerpo desde el ejercicio constan te para conocerlo y controlarlo.

La tarea de la enseñanza se le asignó al maestro, quien también fue objeto del saber pedagógico. Dada su importancia y su responsabilidad con la niñez, aquél fue vigilado desde las mismas instituciones que lo contrataron. Entre las conductas que fueron objeto de sanción, estuvieron las faltas cometidas contra el pudor, el aseo, la autoridad y la moderación. Estas fueron penalizadas hasta con la pérdida del empleo. Se supervisó el estado de sus facultades físicas, invitando a realizar ejercicio constante, a mantener dietas especiales y baños necesarios para la salud, todo lo cual debía combinarse con el estudio y la lectura. En cuanto a las facultades morales, su vigilancia fue estricta para garantizar la castidad de los maestros con sus alumnos. Se les vigiló también en su vida cotidiana, en su comportamiento diario en la calle y en la iglesia. Ante todo debió comportarse con total rectitud, y en lo que tuvo que ver con sus facultades intelectuales, se le conmino a estudiar permanentemente lo que competía a su enseñanza, los contenidos, los métodos, lo concerniente al niño y las distintas etapas de su desarrollo. Tuvo, pues, entre otras, la tarea de modelar el carácter infantil, su enseñanza y su gobierno. Con tanta responsabilidad, el maestro fue objeto por parte del Estado), de su preparación en escuelas normales, que dirigían su educación hacia los fines que la sociedad se proponía: formar al maestro para cumplir sir labor de apóstol de la enseñanza.

\section{De cómo nos constituimos en sujetos desde la práctico pedagógica}

El proyecto civilizador, para hacerse realidad tuvo que construir desde sí mismo el tipo de sujeto que debía servirle a sus fines modernos y progresistas. Este proyecto civilizador estuvo en estrecha y dependiente relación con la religión católica, la patria y el progreso. Un proyecto que se justificaba desde la decadencia de la raza colombiana que venía anunciándose. Pero, ¿cómo progresar con moralidad?, pues vigilando a los hombres y educando a los niños desde su debilidad, su carencia, su fragilidad y su volubilidad, por el camino del bien y modelando su cuerpo y su alma hacia los ideales nacionales. Las estrategias de las que se sirvió el poder desde la práctica pedagógica, fueron las mismas que utilizaba el catolicismo. Entre ellas, las prácticas confesionales, que privilegiaron la renuncia de sí mismo como principio de salvación y convirtieron el conocimiento de sí mismo en herramienta de autocontrol.

La moral se entendió desde los preceptos religiosos y se hizo desde entonces inseparable de las cualidades, características y deberes del católico; de ahí que se justificara el papel del clero y de las órdenes religiosas en la enseñanza religiosa en las escuelas, como condición de educación, progreso y civilización. Entre sus presupuestos estuvieron el de combatir entre los niños y jóvenes la influencia de las pasiones y malas inclinaciones, el de educar en los buenos y bellos sentimientos, e inculcarles buenos hábitos y formas adecuadas de comportarse por medio de la enseñanza de las virtudes. Para ello, se utilizaron las prácticas corporales en la escuela, lugar de convergencia de las múltiples fuerzas de poder y de saber, todas ellas construyendo un conocimiento alrededor del cuerpo infantil. Entonces, el cuerpo participó activamente en el desarrollo armónico de las facultades físicas y morales y facilitó con su acción el desarrollo de las facultades intelectuales de los niños. 


\section{Prácticas corporales escolares}

Para alcanzar las virtudes católicas, tan necesarias y anheladas, la educación se sirvió de hábitos corno la obediencia y la atención, necesarias para su correcto aprendizaje. Entre las virtudes que se enseñaron a través de prácticas corporales durante la infancia estuvieron: el orden, el trabajo, la disciplina, la higiene y la castidad.

En cuanto al orden, considerado la base de todo régimen escolar, se pusieron en marcha prácticas corporales en el tiempo, el espacio, el mobiliario escolar y la postura corporal. Todo debía estar en su lugar en el momento justo. Se cumplieron entonces horarios, ejercicios repetidos, movimientos sincronizados del cuerpo, formaciones, respuesta rápida a las ordenes recibidas por los maestros. Se organizó el tiempo de los descansos, de la comida, del sueño y de toda actividad. Así, el tienipo como la savia de la vida, tomó fuerza marchando al lado del progreso. Se prohibió perder el tiempo, y se castigó ese pecado. Los espacios fueron reglamentados en sus dimensiones y el mobiliario en su tamaño, altura, cercanía y posición, con respecto al niño sentado o de pie en el salón de clase.

En lo que respecta a la postura del cuerpo, las prácticas escolares se dirigieron a prevenir lesiones óseas y musculares, a formar una actitud moral de respeto, obediencia y diligencia frente al adulto, y a educar en las buenas maneras no solo en la escuela sino fuera de ella.

El trabajo, virtud que dignifica e impele hacia adelante a las naciones y que garantiza placer por lo adquirido desde el esfuerzo pulsó y organizo en la escuela desde la idea del valor personal. A partir de las obras, al hacer que el niño trabajara, se alejó de la malicia y de los vicios y se hizo útil a la sociedad. De allí que la urgencia fuera mantener a la infancia ocupada todo el tiempo y siempre en actividades que tuvieran utilidad y sentido. Así, al trabajo se llegó desde el juego, por ello, la escuela fue el taller para aprender a trabajar.

La disciplina, virtud necesaria en el ordenamiento de la conducta moral infantil desde los parámetros de los adultos -el deber ser-, supuso la instauración de una pedagogía de la obediencia, y para su debido cumplimiento se acudió al uso del castigo, para encauzar, y al uso de la recompensa, como prefijo. Se llegó a considerar y a tratar a los niños desobedientes como infractores y criminales cuyas penas se asimilaron en muchos casos a las infligidas en las cárceles. El cuerpo fue, entonces, objeto de castigo; se le golpeó y privó de alimento y descanso, y se le sometió a posiciones extremas y dolorosas que atentaron en muchos casos contra su salud. Se castigó al niño y también al maestro. Se le vigiló desde todos los lugares y momentos, situación que hizo difícil escapar a las sanciones por leves que fueran las faltas.

La virtud de la higiene del alma, para el siglo XIX, que consistió inicialmente en abstenerse de todo lo malo, evitando los excesos y siendo temperante en todas las cosas, se vio influida por la búsqueda de la salud desde el saber de la medicina, asunto que entro con tuerza en el espacio educativo. Se trato, entonces, no sol o de la limpieza; sino también de una serie de preceptos encaminados a garantizar la salud y a evitar las enfermedades. A ello contribuyo la revisión y supervisión de los espacios escolares donde los niños permanecían por largo tiempo: se pretendió controlar la temperatura, la iluminación, la ventilación y la comodidad para el movimiento infantil. 
Aunque no faltaron los discursos sobre la castidad de hombres y mujeres en donde la pureza de costumbres fue la virtud por excelencia de la juventud, esta fue una virtud particularmente femenina. Los niños, se afirmó, traían consigo una serie de predisposiciones morbosas, morales y físicas al nacer, mucho mas acentuadas en las niñas; de allí que la educación tuviera que luchar contra lo nocivo de ellas. Debía protegerse, ante todo, la inocencia infantil. Su secreto consistió en abstenerse en doblegar el carácter desde la razón y en controlar el cuerpo ejerciendo dominio estricto sobre él. Se enseñó el autoexamen como la forma para conocer las flaquezas y obrar sobre ellas. Se insistió en la obediencia total a las leyes sociales impuestas y ejercidas en su nombre por los padres, los maestros, los hermanos o los esposos, pues todos al mismo tiempo tuvieron la misión de arrancar del alma de las niñas y mujeres la pereza, la voluptuosidad, el vicio y los malos pensamientos. Se buscaba, finalmente, educar desde las prácticas corporales, en el decoro, la pureza, la obediencia y la prudencia, como herramientas femeninas que le permitieran luchar contra los placeres, los vicios y las tentaciones del mundo moderno.

La urbanidad, entendida como la suma total de las virtudes de un buen ciudadano, constituidas por las formas exteriores del hombre en sociedad, dieron cuenta de un interior limpio y puro. Así, el cuerpo actuó como el cristal a través del cual podía mirarse. Como el arte de la imitación, la urbanidad recogió las normas socialmente aceptadas, dando cuenta del deber ser. Los manuales acerca de esta práctica escolar, recogieron todo lo referente a la higiene, la disciplina, la obediencia y la castidad. Normas y reglas que tuvieron que aprenderse y aplicarse y de lo cual se encargó, también, el maestro. La urbanidad obligó a observar cuidadosamente el cuerpo infantil, para erradicar inmediatamente cualquier incorrección. De esta forma, penetró todos los espacios, todos los tiempos y todas las prácticas en las que el cuerpo actuó.

En la educación del sentimiento infantil, como con las virtudes y la urbanidad, se acudió a la repetición y al hábito. Los buenos sentimientos tuvieron su relación directa con el sentido de lo bello, una posición estética desde la moral católica. Así, el sentimiento de lo bello supuso la elección del orden como el poder de la invención y de la razón. Un orden que se logró desde prácticas corporales ya enunciadas.

\section{La educación física una nueva tecnología educativa}

La recurrencia de prácticas corporales escolares, el saber de la Educación física, que llegó desde Europa a través de manuales y libros, y la preocupación de la educación desde sus facultades, herencia católica y pestalozziana, entre otras, hicieron posible la aparición de la Educación física en la escuela como una rama de instrucción. Entre sus beneficios educativos, estaban el desarrollo de las virtudes morales, el apoyo a la educación intelectual por medio del descanso mental con actividades físicas y el desarrollo físico de la infancia.

El conocimiento, la discusión y la experimentación alrededor del entrenamiento físico en Europa desde finales del siglo XVIII y su influencia en la Educación física colombiana, hace su entrada en el siglo XX, por la década del treinta, aproximadamente. Mientras esto sucede, algunos conceptos y elementos teóricos fueron modificando las prácticas corporales hacia la búsqueda de un régimen de vida dosificado, que beneficiaría el adelanto intelectual y el mejoramiento del estado de salud individual, en provecho de un desarrollo armónico de las facultades. Aparece entonces, un saber que se constituye a comienzos del siglo $\mathrm{XX}$, en una nueva tecnología educativa. 
Aquí, el cuerpo, lugar de conversión de energías, fue objeto de prácticas con el objetivo de alcanzar el equilibrio de sus fuerzas. De ahí la preocupación de la Educación física por la incidencia de los factores externos e internos en la educación del niño y el joven y su afán por controlar y dosificar su actividad corporal en el tiempo y en el espacio.

La propuesta pedagógico arquitectónica de organizar el cuerpo como una máquina que tenía movimientos precisos, un cuerpo con gestos y actitudes medidas y ordenadas rítmicamente y que debía dar paso a una pedagogía escolar que mejorara los rendimientos -ya no la arquitectura, si la ingeniería corporal-, se vivieron simultáneamente, es decir, que estas tendencias penetraron las prácticas corporales sin distinción, juntas, apuntando esencialmente a contribuir a la educación armónica del niño moderno.

La máxima satírica del romano Juvenal, del siglo I, "mente sana en cuerpo Sano, que recorre todoel período de investigación, se tomo descontextualizada de su época, adjudicándosele preceptos cristianos distintos del espíritu que la animó. Con esta frase asistimos al principio constitutivo del hombre por el cristianismo, un ser dual integrado por cuerpo y alma, hecho a imagen y semejanza de Dios y dependiente e independiente a la vez de la divinidad. En esta máxima se apoyó la construcción de la Educación física y moral de ese tiempo, un tiempo católico desde fines políticos y económicos, en donde el trabajo como virtud cristiana, necesitó cada vez más de un cuerpo apto, productivo y bueno, como sostén del alma que seria por ello, también buena.

El lugar de la Educación física hasta 1912, aunque de gran ayuda para la educación moral y la educación intelectual, fue inferior, ya que su objeto, que era el cuerpo, ocupo frente al alma un segundo lugar en importancia, más no en su conocimiento y su control, determinantes a la hora de albergar el alma que se quería. Ya en 1913 discursos como el de Jiménez López, apuntaron a igualar el valor de la educación del cuerpo con la educación de la inteligencia y de la voluntad.

La Educación física, en el primer momento, debió robustecer antes que afeminar, en condiciones precarias antes que complacientes, fortaleciendo tanto el intelecto como los músculos; todo esto para que el alma no se sintiera atormentada y presa entre un cuerpo débil e incapaz. Para el segundo momento, en cambio, la preponderancia de los fines morales sobre los fines físicos, de las prácticas corporales, empezará a cambiar lentamente; será entonces una nueva fuerza, otro poder, otro saber, que atravesará el cuerpo va no sólo para favorecer el aseo y la higiene sino para cuidar de la salud, ahora social, como un necesario e inmediato beneficio a la producción industrial que se le asigna a toda sociedad moderna y progresista. Este auge que comienza a tomar la medicina sobre el cuerpo, no desplazará a la moral, al contrario, será reforzada y por consiguiente generara una mayor sujeción del cuerpo a más saberes.

La preocupación por la salud a finales del siglo XIX, fue más del orden de la higiene como práctica de aseo. Ella buscaba en cada niño, inculcarle hábitos como el baño, por lo menos semanal; el cambio de ropa permanente; y la limpieza de manos y cara. Sin embargo, el discurso que aparece alrededor de la década de 1870, y que afirmaba cómo la formación del ciudadano debía Contener normas que garantizaran una salud adecuada para afrontar los retos del futuro en cuanto a la lucha por la vida y el rendimiento en el trabajo, van evidenciando desde su práctica ciertos problemas que harán posible la intervención y participación del médico en el espacio escolar. Este discurso propendía por 
el equilibrio de las actividades intelectuales y físicas en la escuela, recomendaba el ejercicio físico como remedio a la fatiga mental y le daba importancia a las recreaciones durante las jornadas escolares.

En las primeras décadas del siglo $\mathrm{XX}$, la presencia del médico en la escuela tuvo que ver, también, con la preocupación por la enfermedad colectiva antes que individual. Así, entre sus actividades estaba recomendar las condiciones necesarias para la buena salud de los niños, en cuanto a los espacios, las actividades y el régimen escolar. Pero, quizás lo más importante, fue que a partir del examen que se realizaba a los alumnos, decidía el tipo de Educación física que cada uno debía recibir según su estado y condición. Con él aparecen registros que dan cuenta de la salud de cada niño, como es el caso del certificado de salud, sin el cual no era posible ingresar a una institución educativa o renovar cada año su entrada. Este poder en manos de los médicos, preocupados, como toda la sociedad, en la regeneración de la raza colombiana, se apoyó y se nutrió de escritos como los del médico Jorge Bejarano, en donde el saber de la Educación física se ubicó al lado de la educación intelectual y moral.

La medicina validó a la cultura física desde las prácticas científicas de la fisiología y la higiene y le cedió su administración escolar al médico. La apreciación y el valor de la vida estaba representada en su utilidad social, así, el cuerpo como instrumento de trabajo, debía ponerse a tono con las exigencias modernas. Por esto, la Educación física debió velar no sólo por la salud de los individuos, sino también por el mejoramiento de la raza, pues era la escuela el lugar en donde se acentuaban los vicios, la herencia y la sedentariedad.

El papel que debió cumplir la Educación física con las mujeres, fuera de la educación moral enunciada arriba, fue el de fortalecer su cuerpo para engendrar una prole sana, pues esta era su misión primordial en la sociedad, que se completaba después en la crianza de los hijos; crianza que necesitaba fuerza, sacrificio y buen estado físico.

Los métodos usados para la enseñanza de este ramo de instrucción, estuvieron encaminados en dos direcciones: una, tuvo que ver con la forma general de la enseñanza; recomendaciones para el maestro acerca de la dirección del aprendizaje, el manejo de los alumnos y el desarrollo de los temas, entre otros; y la otra, se dirigió hacia la manera de enseñar la calistenia, la gimnasia, los juegos, los deportes, como en el caso del método sueco, del método alemán y del método inglés. $Y$ los medios de los que se sirvió la Educación física como saber y ramo de instrucción fueron especialmente la gimnasia, los juegos y los deportes. La gimnasia, que en muchos casos compartió los fines de la Educación física, pues se le consideró su igual, fue a la vez un medio para fortificar y dar soltura a los movimientos y un método para llevar a feliz término los propósitos que esta educación se había propuesto. Como método sueco, no se le asignó a la infancia sino a la juventud, por privilegiar el desarrollo particular de los órganos y músculos sobre el desarrollo general de todo el cuerpo. Los, juegos por su parte, se dividieron en dos; por un lado, se impulsó el juego libremente realizado por los niños, como la forma más natural y completa de desarrollarse físicamente; y por el otro, fueron los juegos desde entonces dirigidos por el maestro para alcanzar fines que él se había propuesto con anterioridad: aprendizaje de oficios, formas superiores al juego como el deporte, normalización de la actividad infantil y homogeneización de los ritmos escolares. Los deportes, en cambio, fueron enseñados con preferencia en niños mayores, pues se afirmó que, dada su complejidad y su efecto sobre el funcionamiento del cuerpo, sólo podían enseñarse cuando los jóvenes hubiesen alcanzado la madurez suficiente para no perjudicar su salud. 
La reglamentación de la Educación física, fue permanente tanto a nivel nacional como a nivel regional e institucional. Se habló de su obligatoriedad, necesidad, fines, planes y programas; de su lugar en el tiempo escolar, como ramo de instrucción, de los profesores que la enseñaron; también se discutió sobre aspectos importantes para la época, como la gimnasia, la higiene y las evoluciones militares; se reglamentó y orientó su enseñanza en las escuelas normales, haciendo la distinción entre una enseñanza para mujeres y una para hombres en cada uno de los niveles escolares que tuvo el sistema educativo. Los programas, en especial, fueron difundidos entre los profesores a través de los manuales escolares de la época, que para el caso colombiano fueron dos importantes publicaciones: el periódico La Escuela Normal de Bogotá y la Revista de Instrucción Pública de Colombia, que aparecen durante el periodo de investigación, de forma permanente. Además de estos manuales, que fueron dados a conocer en diferentes entregas, circularon algunos libros que contenían los ejercicios que debían realizarse con especificación de la cantidad, el tiempo de ejecución y la forma. Estas explicaciones se acompañaban de ilustraciones que, además de apoyar lo dicho, mostraban el modelo corporal que debía buscarse por medio de la práctica permanente del ejercicio físico.

Así, el maestro de escuela, debió velar, tanto por el estado general del cuerpo; -el estado particular de sus órganos por medio de una actividad sin tregua, científicamente realizada, que le brindara la voluntad para luchar contra las pasiones e instintos- como por la fuerza física, la disciplina, la salud en el trabajo y el perfeccionamiento en todos los aspectos. Por ello fue urgente su preparación en todo aquello que favorecía a la enseñanza.

De esta forma, se dio comienzo a un capítulo en la educación, en el que fue imposible abandonar la enseñanza de la Educación física dentro y fuera de la escuela, además de otros efectos, por sus beneficios médico-fisiológicos sobre los organismos en desarrollo. Una nueva fuerza, un nuevo saber, hizo su aparición en el inmenso abanico de influencias que penetraron el cuerpo infantil, moldeándolo y controlándolo. La Educación física mejoraría la raza colombiana y la haría más útil, mas productiva, menos enferma, más bella y más buena.

\section{Referencias}

ALVAREZ, Alejandro, (1995). Y la escuela se hizo necesaria, Bogotá,-Colombia, Editorial Magisterio, Págs. 7-11.

BARREAU, Jean Jaques; MORNE, Jean jaques, (1991). Epistemología y antropología del deporte, Madrid-España, Alianza Editorial, 1991.

CANETTI, Elías, (1981). Masa t poder, $3^{\text {a }}$ Edic., Madrid España, Muchnik Editores, SA.

CASTILLA, C., (1872-1873). Perder el tiempo, en El álbum de los niños, Tunja Colombia, serie I, No. 1-24.

DONZELOT, Jacques, (1977). La policía de las familias, en Pretextos, Valencia España.

FOCAULT, MIchel, (1983). Historia de la sexualidad, 9a. Edic., Colombia, Siglo XXI Editores. 
Ediciones la Piqueta.

(1990). La vida de los hombres infames, Madrid - España,

Ediciones la Piqueta.

(1992). Microfísica del poder. $3^{a}$ edc., Madrid - España, (1993). Historia de la locura en la época clásica, ts. I-II, Bogotá - Colombia, Fondo de Cultura Económica.

FRAISE, P., (1976). Psicología del ritmo, 1a edic., Madrid - España, Ediciones Morato SA., pgs. 20-21, 80, 97-98, 103-104.

GUARÍN, Romualdo, (1874). Guía de institutores, en La Escuela Normal, Bogotá Colombia, t. V, Pág. 238.

LLERAS, Martín, (1872). Pedagogía, en La escuela Normal, Bogotá - Colombia, t. III, Págs. 309,310.

NARADOWSKI, Mariano, (1996). Pedagogía y Epistemología, en Tendencias y retos (Coloquio Internacional Educación y Pedagogía Contemporáneas), Bogotá - Colombia.

PROCULTURA SA., Instituto Colombiano de Cultura, (1982).

Manual le historia dc Colombia, $2^{\mathrm{a}}$ edic., t. III.

RAUCH, Andre, (1985). El cuerpo en la educación tísica, II Buenos Aires-Argentina, Editorial Kapelusz. 1981.

SFNOSIAIN, Serafín, (1981). El Cuerpo Tenebroso, en Pretextos, Valencia - España,

SMILES, Samuel, (1981). El Caracter; en Anales de la instrucción publica de los estados unidos de Colombia, Bogotá-Colombia. T. II, núms. 7-11. 